\title{
Ecofisiología del cultivo de la papa (Solanum tuberosum L.)
}

\author{
Ecophysiology of the potato \\ (Solanum tuberosum L.) crop
}

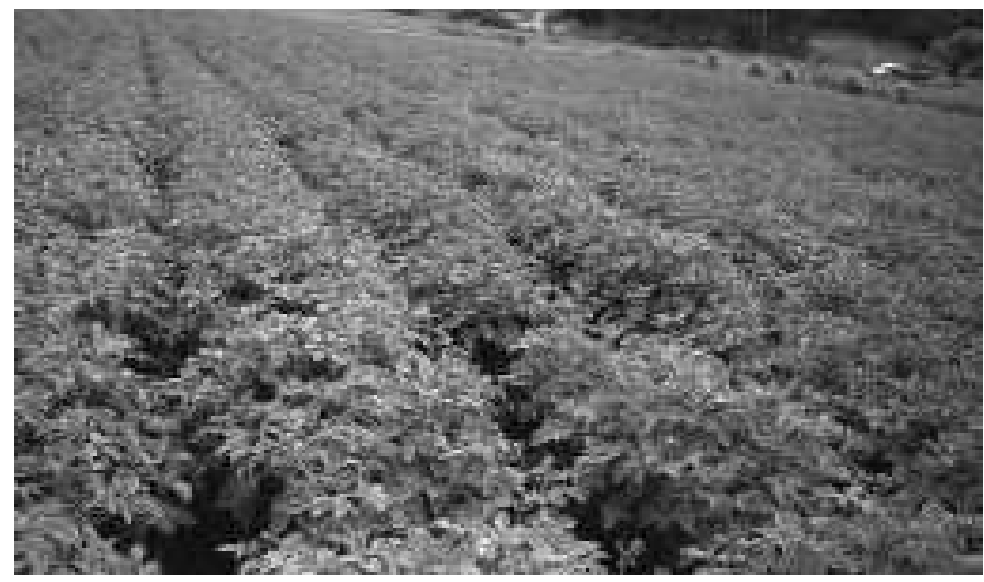

Cultivo de papa en la vereda El Tablón de Táquira, Simijaca, Cundinamarca.

Foto: L. Rodríguez-Pérez

\section{RESUMEN}

La papa es uno de los cinco productos agrícolas de mayor consumo humano en el mundo; su planta es una dicotiledónea perteneciente a la familia de las Solanáceas, y se produce en regiones de latitudes entre los $47^{\circ} \mathrm{S}$ y los $65^{\circ} \mathrm{N}$. El 50\% del área global del cultivo de papa está localizado por encima de los 1.000 msnm, y la zona óptima de producción para las variedades comerciales de Solanum tuberosum está en altitudes ubicadas entre los 2.500 y los $3.000 \mathrm{msnm}$. En cuanto a radiación, niveles por encima de los $1.200 \mu \mathrm{moles} \mathrm{m}^{-2} \mathrm{~s}^{-1}$ generan efectos positivos en la distribución de materia seca hacia los tubérculos. El fotoperiodo corto (10 horas luz) acelera el inicio de la tuberización en la mayoría de variedades. El cultivo de papa se adapta a climas fríos tropicales con temperaturas medias entre 15 y $18^{\circ} \mathrm{C}$, en el suelo y en el aire. La utilización de recursos hídricos y minerales del suelo y de recursos de la atmósfera, como $\mathrm{CO}_{2}, \mathrm{O}_{2}$ y radiación fotosintéticamente activa (RFA), son factores limitantes para el crecimiento y desarrollo de la planta de papa.

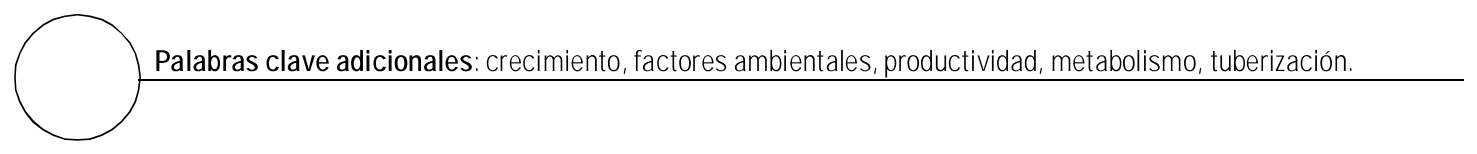

\footnotetext{
1 Departamento de Biología, Pontificia Universidad Javeriana, Bogotá (Colombia). Ioyla.rodriguez@javeriana.edu.co
} 


\begin{abstract}
The potato crop is one of the five most consumed products by humans. The potato is an herbaceous dicotyledonous plant belonging to the Solanaceae family, allowing crop establishment and production within the latitudes $47^{\circ} \mathrm{S}$ and $65^{\circ} \mathrm{N}$. Of the total global surface area occupied by the potato, $50 \%$ is located above $1,000 \mathrm{~m}$ a.s.I., and the optimal zone of production of Solanum tuberosum of the commercial varieties is between 2,500 and $3,000 \mathrm{~m}$ a.s.l. As far as radiation, levels above 1,200 micromoles $\mathrm{m}^{-2} \mathrm{~s}^{-1}$ provoke positive effects on the dry matter distribution in tubers. A short photoperiod (10 hours of light) accelerates the start of tuberization in most commercial varieties. The potato crop adapts to cold tropical climates with soil and air mean temperatures between 15 and $18^{\circ} \mathrm{C}$. Soil resources, atmospheric resources, such as $\mathrm{CO}_{2}, \mathrm{O}_{2}$ and photosynthetically active radiation (PAR) are limiting factors to plant growth and development.
\end{abstract}

Additional key words: growth, environmental factors, productivity, metabolism, tuberization.

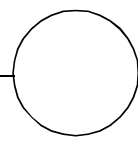

Fecha de recepción: 13-01-2010

Aprobado para publicación: 02-06-2010

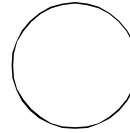

En el mundo entero, la papa se ha consolidado como el alimento esencial de muchas civilizaciones (Estrada, 2000; Brown, 1993), debido a sus características nutricionales, por ello, constantemente es objeto de múltiples investigaciones. En la composición nutricional de la papa se destaca la presencia de carbohidratos, proteínas, vitaminas (riboflavina, niacina, vitamina A y C, tiamina, ácido fólico, ácido pantotéico) y minerales (hierro, calcio, fosforo, magnesio, zinc). En la alimentación humana, la papa se consume fresca o procesada; la fresca constituye el renglón más importante, como destacada fuente de almidón y otros carbohidratos (FAO, 2009).

El presente artículo hace una revisión de la ecofisiología del cultivo de la papa, destacando aspectos relevantes como origen y distribución, caracterización y factores agroecológicos de importancia para el cultivo.

\section{ORIGEN Y DISTRIBUCIÓN}

Los estudios históricos reportan los tubérculos de papa como la base alimentaria de las comunidades andinas precolombinas. La denominación «tubérculos» fue dada por los indígenas aymarás y quetchuas. La mayor variabilidad genética de papa se reporta para Perú y Bolivia, alrededor del lago
Titicaca, de donde se extendió a Chile, Ecuador y Colombia, países en los que fue cultivada por las comunidades chibchas (Brown, 1993; Spooner et al., 2005). En el continente europeo, el primer registro es para el año de 1573, en Sevilla (España) este indica que los tubérculos se utilizaban en la alimentación. El cultivo de papa fue introducido desde España a otros países europeos, como Italia, en 1596; Inglaterra y Alemania, en 1601 (Brown, 1993; Estrada, 2000).

La producción de tubérculos de papa ocupa el cuarto lugar en el sistema de alimentación mundial. Según estadísticas de la FAO (2009), la producción mundial de papa para el año 2009 fue de 309,3 millones de toneladas. La producción por continentes se presenta en la tabla 1. En Colombia, de acuerdo con las estadísticas del Ministerio de Agricultura y Desarrollo Rural, para el año 2009, el área cultivada de papa fue de 123.659 ha año-1, $^{-1}$ con una producción de 2.272.772 t.

\section{CARACTERIZACIÓN DE LA PLANTA DE PAPA}

\section{Morfología}

Las plantas de la especie Solanum tuberosum L. pertenecen a la familia de las Solanáceas (Spooner et al., 2005); son dicotiledóneas herbáceas, con 


\begin{tabular}{|l|c|c|c|}
\hline \multicolumn{4}{|l|}{ Tabla 1. Producción mundial de papa por continentes. } \\
\hline \multicolumn{1}{|c|}{ Continente } & Área cosechada (ha) & Producción ( $\mathrm{t})$ & Rendimiento $\left(\mathrm{t}_{\text { ha }}{ }^{ }\right.$) \\
\hline África & 1.541 .498 & 17.05 .500 & 11.1 \\
\hline Asia & 8.751 .259 & 143.259 .252 & 16,3 \\
\hline Oceanía & 44.402 & 1.671 .602 & 3.7 \\
\hline Europa & 6.275 .549 & 123.817 .694 & 19,7 \\
\hline América & 1.549 .532 & 40.644 .974 & 26,2 \\
\hline Mundo & 16.620 .742 & 309.393 .522 & 12,4 \\
\hline
\end{tabular}

Fuente: FAO (2009)

un sistema radical fibroso, originado a partir de los primordios radicales de los brotes que emergen del tubérculo madre (Lujan, 1991; Allen, 1978; Lujan, 1994; Tekalign y Hammes, 2005). Los tallos aéreos de la planta se originan directamente del tubérculo madre, y los secundarios o ramificaciones crecen de las yemas axilares de los nudos, de los tallos principales; los estolones son tallos que se originan de las yemas axilares de los nudos de tal los principales subterráneos, que posteriormente desarrollarán los tubérculos o tallos de almacenamiento (Lujan, 1991; Allen, 1978; Lujan, 1994; Tekalign y Hammes, 2005). Las hojas son compuestas, alternas e insertas a los nudos del tallo en disposición espiral; el raquis es la parte central de la lámina, sobre el cual se desarrollan los foliolos y pseudoestípulas, cuyas funciones involucran la absorción de radiación fotosintéticamente activa (RFA) y la incorporación de $\mathrm{CO}_{2}$ para el proceso de fotosíntesis foliar (Allen, 1978; Lujan, 1994; Tekalign y Hammes, 2005). Las flores son bisexuales con cáliz, corola, androceo y gineceo, que en conjunto forman una inflorescencia cimosa. Los frutos son bayas con numerosas semillas de importancia para la reproducción sexual de la especie (Lujan, 1991; Allen, 1978; Lujan, 1994).

\section{Estados fenológicos}

Los estados fenológicos de S. tuberosum L., se presentan en la tabla 2. Las plantas de papa se establecen sembrando tubérculos semilla, a partir de los cuales crece uno o múltiples brotes, dependiendo de la dominancia apical. En el estado de brotación, los carbohidratos almacenados en los tubérculos son la principal fuente para el crecimiento de tallos aéreos. Paralelamente al crecimiento de los brotes, en el desarrollo de partes vegetativas, ocurre la formación de las hojas, inicialmente desde primordios foliares hasta el desarrollo de hojas completas; el crecimiento foliar continúa durante el ciclo biológico hasta que las plantas alcanzan la madurez fisiológica (Allen, 1978; Sonnewald, 2001; Meier, 2001; Vreugdenhil et al., 2007). Con el establecimiento de las plantas ocurren discretos procesos fisiológicos de síntesis, translocación y acumulación de materia seca en los órganos de la planta.

Durante el desarrollo de las partes vegetativas cosechables, los estolones inician la acumulación de almidón en un localizado abultamiento ubicado entre el octavo y el duodécimo entrenudo desde el ápice del estolón. Los entrenudos sucesivos de la punta del estolón crecen longitudinal y radialmente por división y expansión celular, hasta alcanzar el crecimiento completo de los tubérculos. Los puntos de crecimiento (ojos) de los tubérculos están arreglados en filotaxis espiral y de estos se originan (Menzel, 1985; Lujan, 1991; Lujan, 1994; Sonnewald, 2001; Meier, 2001). En estado de llenado de los tubérculos, la distribución de fotoasimilados desde las hojas fotosintéticas asegura la formación de los tubérculos. Cuando el Ilenado de tubérculos ha finalizado, se presentan discretos cambios a nivel bioquímico y anatómico, incluyendo el desarrollo de la cáscara o peridermis (Menzel, 1985; Lujan, 1994; Sonnewald, 2001; Meier, 2001). Durante el almacenamiento, después de la cosecha, los 
Tabla 2. Estadios principales de desarrollo de la planta de papa $S$. tuberosum L., según escala BBCH.

\begin{tabular}{|c|c|}
\hline Estadio principal & Descripción \\
\hline Germinación/Brotación & $\begin{array}{l}\text { Inicio de la formación de brotes, tallos y raíces. Formación y desarrollo } \\
\text { de los estolones. Los tallos traspasan la superficie del suelo. }\end{array}$ \\
\hline Desarrollo de las hojas & Las hojas adquieren más de $4 \mathrm{~cm}^{2}$ de área foliar. \\
\hline Formación de brotes laterales & Formación de brotes basales laterales visibles. \\
\hline Crecimiento longitudinal (Brotes principales) & $\begin{array}{l}\text { Comprende de comienzo a finalización de la cobertura del cultivo. Las } \\
\text { plantas se tocan entre hileras. }\end{array}$ \\
\hline Desarrollo de las partes vegetativas cosechables & $\begin{array}{l}\text { Comienzo de la formación y llenado de tubérculos, alcanzando la máxima } \\
\text { masa final total. La peridermis madura. }\end{array}$ \\
\hline Aparición del órgano floral & Formación de las inflorescencias de la planta. \\
\hline Floración & Flores en antesis, floración plena del cultivo y fin de la floración. \\
\hline Formación del fruto & Formación de bayas. \\
\hline Maduración de frutos y semillas & Desarrollo y maduración de bayas y semillas. \\
\hline Senescencia & $\begin{array}{l}\text { Amarillamiento de hojas, posteriormente se tornan de color marrón. } \\
\text { Hojas y tallos muertos, blanquecinos y secos. Momento para la } \\
\text { cosecha. }\end{array}$ \\
\hline
\end{tabular}

Fuente: Meier (2001).

tubérculos inician el periodo de dormancia; solamente unas semanas después se reanuda el crecimiento de brotes, cuando la dormancia ha finalizado. El porcentaje y el modelo de crecimiento de yemas están determinados por la duración y la temperatura de almacenamiento (Menzel, 1985). Al final del ciclo ocurre la floración de las plantas y la reproducción sexual, que conducen a la formación de semillas viables. Cuando el cultivo de papa alcanza la madurez fisiológica, ingresa en senescencia; en este estado, los órganos de la planta adquieren una apariencia seca y blanquecina, que indica la pérdida de clorofila, del RNA y las proteínas (Allen, 1978; Allen y Scott, 1980).

\section{FISIOLOGÍA DE Solanum tuberosum L.}

El crecimiento, desarrollo y rendimiento del cultivo de papa dependen de la interacción del genotipo de la variedad y los factores agroecológicos (Allen, 1978). Para producir rendimientos de óptima calidad, Ias plantas requieren un adecuado suministro de recursos del suelo (agua y nutrientes minerales) y recursos de la atmósfera, como $\mathrm{CO}_{2} \mathrm{O}_{2}$ y RFA (Tekalign y Hammes, 2005; Larcher, 2003; Vreugdenhil et al., 2007). Más del $90 \%$ de la materia seca de una planta de papa se sintetiza durante el proceso de fotosíntesis, en el cual, el $\mathrm{CO}_{2}$, fijado y reducido a carbohidratos, es determinante para la producción de tubérculos y otros órganos vegetales. La planta de papa presenta vía fotosintética C3 (Mackerron y Waister, 1983; Dwelle et al., 1983). En general, el cultivo de la papa desarrolla un follaje de larga duración, que favorece la intercepción de una alta proporción de RFA (Lujan, 1994; Dwelle et al., 1983). Las longitudes de onda utilizadas por el aparato fotosintético son aquellas ubicadas entre los 400 y los 700 nm (RFA) (Azcon-Bieto y Talón, 2008). En cultivos de papa se ha encontrado que el Índice de Área Foliar crítico (IAF) debe ser cercano a 3, valor requerido para interceptar de 90 a 95\% de la RAF (Mackerron y Waister, 1983; Burke, 2003; Wu et al., 2007).

En el contexto de la planta como individuo, la relación de fuentes y demandas también tiene la capacidad de afectar las tasas de fotosíntesis; si se incrementan los órganos demanda, se 
incrementa también la actividad de las fuentes (Nosberger y Humpries, 1965; Conn y Cochran, 2006; Burke, 2003; Taiz y Zeiger, 2006). Una vez se ha iniciado la tuberización, la demanda por asimilados se incrementa para el llenado de los tubérculos (Norsberger y Humpries, 1965; Alisdair et al., 2002; Alisdair y Willmitzer, 2001; Burke, 2003).

En la planta de papa, las condiciones ambientales óptimas (RFA, temperatura, agua y nutrientes minerales) influyen de manera directa en la fotosíntesis; la luz influencia la apertura de los estomas y, por lo tanto, la fijación de $\mathrm{CO}_{2}$; la temperatura óptima para una alta captación de $\mathrm{CO}_{2}$ es cercana a $24^{\circ} \mathrm{C}$. La nutrición deficiente, reduce las tasas fotosintéticas (Hay y Walker, 1989; Burke, 2003).

Cuando las plantas de papa están creciendo y al inicio de la tuberización, muchos de los asimilados son utilizados para el desarrollo de órganos vegetativos diferentes a los tubérculos (Alisdair y Willmitzer, 2001; Burke, 2003). Siete días después de iniciada la tuberización, únicamente del 5 al 20\% de los fotoasimilados son translocados a los tubérculos. Después de la tercera semana de iniciada la tuberización, los fotoasimilados son translocados hacia los tubérculos principalmente. Para el final del ciclo biológico, el 95\% de la materia seca es translocada para Ilenado de los tubérculos (Gawronska et al., 1984; Burke, 2003; Vreugdenhil et al., 2007).

El flujo de carbono a través del floema de la planta de papa se divide en cinco fases (Dwelle, 1990; Alisdair y Willmitzer, 2001; Burke, 2003). La primera es la fijación y reducción de carbono o fotosíntesis, proceso que ocurre en el mesófilo de las hojas. La segunda es la asignación del carbono fijado dentro de la hoja entre azúcares y almidón; si el carbono es particionado a sacarosa, este carbohidrato está disponible para el rápido exporte desde el tejido foliar hacia los potenciales vertederos; el almidón, por su parte, es acumulado en las vacuolas y puede ser removilizado durante la noche (Burke, 2003); en la tuberización, las hojas fuente exportan gran cantidad de fotoasimilados hacia los tubérculos en formación y Ilenado. La tercera fase es la carga floemática de sacarosa, que ocurre como un proceso activo. La cuarta fase es la translocación de asimilados a larga distancia, conducida por el flujo en masa. La quinta fase es la descarga, suministro y utilización de asimilados por los tejidos y órganos demanda de la planta, principalmente tubérculos, flores y frutos. En general, el flujo de carbono está determinado por el gradiente de sacarosa entre la fuente foliar y la demanda; este determina la dirección y extensión del movimiento de sacarosa (Dwelle, 1990; Alisdair y Willmitzer, 2001; Burke, 2003). La alta concentración de $\mathrm{CO}_{2}$ en el mesófilo (mayor a $500 \mu \mathrm{mol} \mathrm{mol}{ }^{-1}$ ) afecta positivamente el patrón de translocación de materia seca y de producción de los tubérculos, debido a que induce la exportación rápida de asimilados desde las hojas fuente a los tubérculos (Högy y Fangmeier, 2009; Schapendonk et al., 2000).

\section{FACTORES AMBIENTALES}

Morfológica y fisiológicamente, la planta de papa es afectada por factores ambientales como la altitud, la latitud, la temperatura, la duración del día, la intensidad lumínica, la humedad y la fertilidad del suelo. La expresión del genotipo de la especie puede ser modificada debido a la capacidad que posee para alterar el fenotipo cuando las condiciones medioambientales cambian (Ortiz y Hernández, 1986; Vreugdenhil et al., 2007).

\section{Latitud y altitud}

El cultivo de la papa se distribuye geográficamente en un amplio rango de latitudes y altitudes (Hijmans, 2001). En cuanto a la latitud, la papa se produce en regiones entre los $47^{\circ} \mathrm{S}$ y $\operatorname{los} 65^{\circ} \mathrm{N}$; en Suramérica se cultiva en Bolivia, Perú, Brasil, Argentina, Chile y región sur del Ecuador; sin embargo, el mayor porcentaje de tierra cultivada en papa se ubica entre los 20 y los $60^{\circ} \mathrm{N}$ (Walker et al., 1999; Haverkort, 1990; Hijmans, 2001). En cuanto a altitud, el 50\% del área global del cultivo de papa está localizado a altitudes por encima de los 1.000 msnm, en regiones altas tropicales de los Andes, África (Central y Este), Etiopía e Indonesia; en el hemisferio norte, el porcentaje de área sembrada en papa por debajo de los $1.000 \mathrm{msnm}$ se reduce ostensiblemente, debido a que la papa es una 
planta que se adapta a condiciones de clima frío; en el hemisferio Sur existen zonas de producción de papa en tierras bajas en Mozambique, Angola, Brasil y la costa del Perú.

Entre los $32^{\circ} \mathrm{N}$ y $\operatorname{los} 42^{\circ} \mathrm{N}$ (zona central de China), el área de cultivo de papa está por encima de los 1.000 msnm, y puede ser cultivada en invierno, primavera, verano u otoño (Haverkort, 1990; Walker et al., 1999; Hawkes, 1997; Hijmans, 2001; FAO, 2009). En Colombia, la producción de papa se concentra en regiones de montaña, de clima frío, ubicadas entre los 2.000 y 3.500 msnm, mientras que a alturas inferiores o superiores se reduce el rendimiento; la zona óptima de producción para las variedades Solanum está entre los 2.500 y 3.000 msnm (Haverkort, 1990; Hijmans, 2001).

\section{Viento}

El movimiento de las corrientes de aire tiene importantes efectos sobre el crecimiento, desarrollo y metabolismo del cultivo de papa; el viento puede generar incrementos en las tasas de transpiración, que las hojas y tallos se lesionen por su acción, que las hojas presenten lesiones necróticas como resultado del efecto mecánico y el choque entre ellas; las corrientes de aire rápidas y fuertes generan deshidratación, caída de las hojas y estrés hídrico en las plantas, lo que determina reducciones en la fotosíntesis por cierre estomático y bajas tasas de translocación de asimilados; esto reduce, a su vez, el rendimiento (Casierra-Posada y AguilarAvendaño, 2008). Cuando la velocidad del viento es rápida, las hojas Ilegan a malformarse (por doblamiento) en la parte central del limbo; Ios tallos pueden caerse por retorcimiento, causando rompimiento de tejidos y corte del suministro de nutrientes hacia los órganos aéreos y subterráneos, ya sea por xilema o por floema (Pavlista, 2002; Wright y Brooks, 2002)

\section{Radiación y fotoperiodo}

En las plantas de papa, Ia RFA (entre 400 y 700 $\mathrm{nm}$ ) que es interceptada por el follaje depende de las variedades. El desarrollo y acumulación de materia seca en los tubérculos es mayor a radiaciones continuas de $400 \mu$ moles $\mathrm{m}^{-2} \mathrm{~s}^{-1}$ por periodos de $12 \mathrm{~h}$ de irradianza (Wheeler y Tibbitts, 1986; Vreugdenhil et al., 2007). En el tercio superior del follaje en el cultivo de papa, las hojas pueden absorber hasta $1200 \mu$ moles m ${ }^{2} \mathrm{~s}^{-1}$ y dejar pasar la RFA restante a los tercios medio e inferior del follaje para ser aprovechada en estos. Niveles de radiación inferiores a 250 $\mu$ moles $\mathrm{m}^{-2} \mathrm{~s}^{-1}$ de fotones generan efectos negativos en cuanto a la distribución de biomasa (Kooman et ál, 1996; Wheeler y Tibbitts, 1986; Vreugdenhil et al., 2007).

Aunque las variedades de papa presentan diferencias en cuanto a la respuesta al fotoperiodo, en general, el fotoperiodo corto acelera el inicio de la tuberización en la mayoría de estas(Snyder, 1989; Allemann y Hammes, 2006). Los asimilados exportados desde las hojas es mayor en días cortos que en días largos (Lorenzen y Ewing, 1992; Ewing y Wareing, 1978; Vreugdenhil et al., 2007). La percepción del fotoperiodo ocurre en la hoja y es trasmitida a los estolones para la formación de los tubérculos; la señal es transportada a través del floema acropetalamente. La investigación actual muestra que el fitocromo B está involucrado en la inhibición de la tuberización en día largo y en la inducción de la tuberización en día corto (Jackson, 1999; Snyder, 1989; Allemann y Hammes, 2006; Batutis y Ewing, 1982). Niveles reducidos del fitocromo $B$ inhiben la tuberización en fotoperiodo de día largo (Jackson y Prat, 1996). El fitocromo B también parece estar involucrado en el control de la floración, especialmente en plantas de día corto (Jackson y Prat, 1996: Lorenzen y Ewing, 1992; Ewing y Wareing, 1978; Allemann y Hammes, 2006).

\section{Temperatura}

El cultivo de papa se adapta a climas fríos tropicales con temperaturas medias entre $15 \mathrm{y}$ $18^{\circ} \mathrm{C}$ en el suelo y en el aire (Sarquis et al., 1996; Haverkort, 1990; Yuan y Bland, 2004; Vreugdenhil et al., 2007); temperaturas más altas favorecen el desarrollo de follaje y retardan la tuberización (Van-Delden et al., 2000). El estrés por calor determina el desarrollo de un alto número de tubérculos de menor tamaño por planta, baja gravedad específica, bajo contenido de materia seca y peridermis de los tubérculos 
de colores pálidos. A temperaturas superiores a los $30^{\circ} \mathrm{C}$, la fotosíntesis y la tasa de asimilación de carbono se reducen, lo que a la vez determina reducciones en el crecimiento y rendimiento ( $\mathrm{Ku}$ et al., 1977).

La temperatura influencia la relación fuentedemanda, por lo que la tasa de asimilación neta máxima en el cultivo de papa ocurre entre 15 y $18^{\circ} \mathrm{C}$ (Ku et al., 1977); a temperatura superior $\left(27-28^{\circ} \mathrm{C}\right)$ es mayor la tasa de crecimiento potencial del follaje y, por lo tanto, mayor es su capacidad de consumir asimilados disponibles (Thornton et al., 1996; Lafta y Lorenzen, 1995; Van-Delden et al., 2000). Antes del inicio de la tuberización, la principal demanda de la planta de papa es el follaje; temperaturas de $27^{\circ} \mathrm{C}$ favorecen el crecimiento de ramas y hojas (Ku et al., 1977; Lafta y Lorenzen, 1995; Van-Delden et al., 2000).

Las investigaciones realizadas por Sarquis et al. (1996) reportan que temperaturas superiores a $23{ }^{\circ} \mathrm{C}$ retrasan el inicio de la tuberización, mientras que temperaturas por debajo de $17^{\circ} \mathrm{C}$ reducen la Tasa de Asimilación Neta (TAN) y retardan la tasa de aparición y expansión de hojas para alcanzar una alta intercepción de RFA en corto tiempo. La temperatura óptima para la tuberización está entre 18 y $23^{\circ} \mathrm{C}$.

Los efectos negativos de las heladas (temperaturas del aire entre -2 y $-9^{\circ} \mathrm{C}$ ) en las plantas de papa implican reducción en la absorción de agua por las raíces y en el transporte de agua a nivel celular y de tejidos, reducción en la actividad fotosintética y formación de cristales de hielo en los espacios intercelulares y protoplasto de los tejidos vegetales, que destruyen matrices celulares (Sukumaran y Weiser, 1972).

\section{Nutrición mineral y disponibilidad de agua}

La investigación en nutrición mineral y uso del agua en los cultivos indica que se presentan diferentes respuestas en las especies vegetales (Ulrich, 1993; Walworth y Muniz, 1993: Ribó, 2004; Westermann, 2005; Clarkson, y Hanson, 1980; Vreugdenhil et al., 2007). Para alcanzar una producción óptima, las plantas de papa requieren de $N, P, K, M g, C a, S, Z n, M n, B$ y Cu, y es importante destacar que los genotipos varían en la eficiencia para utilizar y translocar estos nutrientes a los sitios de actividad metabólica (Vreugdenhil et al., 2007). Se puede considerar que para una densidad poblacional promedio de 25.000 plantas/ha y un rendimiento de tubérculos de $50 \mathrm{t} \mathrm{ha}^{-1}$, se requiere por hectárea: $250 \mathrm{~kg}$ de $\mathrm{N}, 92 \mathrm{~kg}$ de $\mathrm{P}_{2} \mathrm{O}_{5}, 360 \mathrm{~kg}$ de $\mathrm{K}_{2} \mathrm{O}, 20 \mathrm{~kg}$ de $\mathrm{Mg}, 25 \mathrm{~kg}$ de $\mathrm{S}, 15 \mathrm{~kg}$ de $\mathrm{Ca}, 6 \mathrm{~kg}$ de Fe, $3 \mathrm{~kg}$ de $\mathrm{Zn}, 2,5 \mathrm{~kg}$ de $\mathrm{Mn}, 2 \mathrm{~kg}$ de $B, 0,5 \mathrm{~kg}$ de Cu y $0,1 \mathrm{~kg}$ de Mo (Hernández, 1989; Ribó, 2004; Westermann, 2005).

La planta de papa es sensible al estrés por déficit hídrico; la disponibilidad de agua en el suelo no puede ser reducida más del 30\% para alcanzar óptimos rendimientos (Darwish et al., 2006; Tourneux et al., 2003a); un suministro adecuado de agua es determinante desde el inicio de la tuberización hasta que la planta alcance la madurez fisiológica para obtener altos rendimientos de excelente calidad (Haverkort et al., 1990; Sermet et al., 2005; Tourneux et al., 2003b). En el cultivo de papa, una alta disponibilidad de asimilados promueve el crecimiento de raíces, estolones, hojas y ramas (Tourneux et al., 2003a, b). Durante la etapa de expansión del follaje, el estrés hídrico reduce la eficiencia fotosintética, el crecimiento del follaje y favorece la partición de asimilados hacia el crecimiento de los tubérculos, sobre todo cuando ya existen tubérculos iniciados en la planta (Haverkort et al., 1990; Sermet et al., 2005; Darwish et al., 2006; Vreugdenhil et al., 2007); otro efecto del estrés hídrico es el acortamiento del crecimiento del follaje (madurez temprana del cultivo) a favor de la partición de asimilados a los tubérculos, lo que determina un acortamiento del ciclo de vida del cultivo (Karafyllidis et al., 1996; Fulai et al., 2006; BaoZhong et al., 2002; Darwish et al., 2006).

La sequía durante el periodo de tuberización y Ilenado de los tubérculos tiene un efecto drástico sobre el rendimiento (Haverkort et al., 1990; Sermet et al., 2005); la disponibilidad limitada de agua durante los diferentes estadios de desarrollo de la papa reduce el crecimiento, el rendimiento, el número de tubérculos por planta y el tamaño y la calidad de estos (Karafyllidis et al., 1996; Dalla Costa et al., 1997; Yuan et al., 2003). 
Así como el estrés hídrico tiene efecto sobre el cultivo de papa, la excesiva irrigación puede generar daños de diferentes formas; por ejemplo, favorece la presencia y severidad de enfermedades, lo que al tiempo reduce el área foliar y la fotosíntesis de la planta, disminuyendo el rendimiento por baja aireación del suelo y escasa respiración en el sistema radical, e incrementando los costos de producción, y resulta en erosión de suelos, al generar lixiviación y contaminación de cuerpos de agua por fertilizantes y productos químicos (Waddell et al., 2000; Fulai et al., 2006; Bao-Zhong, 2003). La deficiente irrigación promueve una reducción en la calidad de los tubérculos y bajos rendimientos debido a reducciones en el área foliar y en la fotosíntesis por unidad de área.

\section{Disponibilidad de $\mathrm{CO}_{2}$}

En los cultivos de papa, la concentración de $\mathrm{CO}_{2}$ también es determinante de la fotosíntesis, productividad y rendimiento. La investigación ha documentado los efectos positivos del incremento en la concentración de $\mathrm{CO}_{2}$ sobre el crecimiento y rendimiento de un amplio rango del cultivos (Kimball, 1983; Vreugdenhil et al., 2007); las plantas de papa expuestas a elevada concentración de $\mathrm{CO}_{2}\left(720 \mathrm{~mL} \mathrm{~L}^{-1}\right)$ por periodos largos incrementan la fotosíntesis entre 10 y $40 \%$, y la acumulación de almidón, en 400\% (Casanova et al., 2005). Las triosas fosfato sintetizadas durante el ciclo de Calvin-Benson son utilizadas para la formación de sacarosa, almidón, otros carbohidratos, aminoácidos, ácidos grasos e isoprenoides. La papa es una especie con capacidad para transportar sacarosa a órganos de almacenamiento (tubérculos). Riesmeier et al., 1994; Farrar y Williams, 1991, y Piikki et al., 2006, reportan un incremento en el rendimiento de tubérculos por elevado $\mathrm{CO}_{2}$; incrementado la concentración de $\mathrm{CO}_{2}$ desde $355 \mathrm{~mL} \mathrm{~L}^{-1} \mathrm{a} 680 \mathrm{~mL}$ $\mathrm{L}^{-1}$ se incrementa en $32 \%$ el peso fresco de los tubérculos. La exposición a elevado $\mathrm{CO}_{2}$ reduce la conductancia estomática, respuesta común para plantas C3 expuestas a esta condición (Farrar y Williams, 1991; Finnan et al., 2002; Conn y Cochran, 2006; Drake et al., 1997; Piikki et al., 2006). Otra respuesta de las plantas de papa frente a niveles elevados de $\mathrm{CO}_{2}$ es el incremento en la concentración de almidón en las hojas
(Conn y Cochran, 2006; Piikki et al., 2006). En general, las plantas de papa expuestas a altas concentraciones de $\mathrm{CO}_{2}$ exhiben respuestas como: incremento en el rendimiento de tubérculos (Donnelly et al., 2001; Piikki et al., 2006), cambio en los patrones de translocación de materia seca (Drake et al., 1997; Finnan et al., 2002), reducción en la conductancia estomática, reducción en la biomasa translocada a tallos, hojas y flores, incremento en tasa de translocación de materia seca hacia los tubérculos e incremento en la relación raíces-brotes (Finnan et al., 2002; Conn y Cochran, 2006; Drake et al., 1997). Los incrementos en las concentraciones de $\mathrm{CO}_{2}$ atmosférico pueden mejorar la calidad de los tubérculos para consumo humano y propósitos industriales, por reducción en el contenido de glico-alcaloides e incremento en el contenido de almidón, aunque el contenido de proteínas también puede reducirse (Donnelly et al., 2001; Piikki et al., 2006; Conn y Cochran, 2006).

\section{CONCLUSIONES}

- Las plantas de papa presentan características morfológicas, fisiológicas y metabólicas que permiten el establecimiento y producción del cultivo en un amplio rango de condiciones medioambientales en diferentes latitudes y altitudes; esto determina su distribución geográfica a nivel mundial en los diferentes ecosistemas, así como su adaptación en diferentes estaciones climáticas.

- Por sus cualidades nutricionales, la papa constituye una fuente básica de la alimentación mundial y es objeto de múltiples investigaciones tendientes a alcanzar mayores logros productivos, traducidos en rendimientos de mejor calidad de acuerdo con los genotipos y sus características.

- La investigación a nivel mundial debe continuar estudiando el efecto de las condiciones ambientales generadas por el cambio climático global sobre el crecimiento, desarrollo y rendimiento de las variedades de importancia económica. 


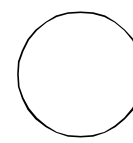

Alisdair, R.; L. Willmitzer y R. Trethewey. 2002. Sucrose to starch: a transition in molecular plant physiology. Trends Plant Sci. 7(1), 35-41.

Alisdair, R.F. y L. Willmitzer. 2001. Molecular and biochemical triggers of potato tuber development. Plant. Physiol. 127, 1459-1465.

Allemann, J. y P. Hammes. 2006. Effect of photoperiod on tuberization in the Livingstone potato (Plectranthus esculentus N.E.Br. Lamiaceae). Field Crops Res. 98, 76-81.

Allen, E. 1978. The potato crop. The scientific basis for improvement. Second edition. Harris and Hall, London.

Allen, E.J. y R. Scott. 1980. An analysis of growth of the potato crop. J. Agric. Sci. 94, 583-606.

Azcón-Bieto, J. y M. Talón. 2008. Fundamentos de fisiología vegetal. Second edition. Interamericana McGraw-Hill, Madrid.

Bao-Zhong, Y.; S. Nishiyama; R. Alisdair; L. Willmitzer y R. Trethewey. 2002. Sucrose to starch: a transition in molecular plant physiology. Trends Plant Sci. 7(1), 35-41.

Batutis, E.J. y E. Ewing. 1982. Far-red reversal of red light effect during long night induction of potato (S. tuberosum L.) tuberization. Plant Physiol. 69, 672-674.

Brown, C.R. 1993. Origin and history of the potato. Amer. Pot. J. 70, 63-373.

Burke, J. 2003. Growing the potato crop. Teagasc. En: Irish Agriculture and Food Development Authority. 3p., http://www.teagasc.ie/ publications/2003/conferences/potato/ paper02.htm; consulta: septiembre de 2010.

Clarkson, D. y J. Hanson. 1980. The mineral nutrition of higher plants. Annu. Rev. Plant Physiol. 31, 239-298.

Casanova, M.A.; G. Hoffmann-Thoma; A.A. Schrier; A. Fangmeier; H. Jager y A. Bel. 2005. Increase of

\section{REFERENCIAS BIBLIOGRÁFICAS}

photosynthesis and starch in potato under elevated $\mathrm{CO}_{2}$ is dependent on leaf age. J. Plant Physiol. 162, 429-438

Casierra-Posada, F. y O. E. Aguilar-Avendaño. 2008 Respuestas fisiológicas y morfológicas de plantas de mora (Rubus sp.) sometidas a estrés por viento inducido. Rev. Colomb. Cienc. Hortic. 2(1), 43-53.

Conn, J. y V. Cochran. 2006. Response of potato (S tuberosum L.) to elevated atmospheric $\mathrm{CO}_{2}$ in the North American Subarctic. Agr. Ecosyst. Environ. 112, 49-57.

Dalla Costa, L.; G. Vedove; G. Gianquinto; R. Giovanardi y A. Peressotti. 1997. Yield, water use efficiency and nitrogen uptake in potato: influence of drought stress. Pot. Res. 40, 19-34.

Darwish, T. M.; T. Atallah; S. Hajhasan y A. Haidar. 2006. Nitrogen and water use efficiency of fertigated processing potato. Agr. Water Manage. 85, 95104.

Donnelly, A.; T. Lawson; J. Craigon; C. Black; J. Colls y G. Landon. 2001. Effects of elevated $\mathrm{CO}_{2}$ and $\mathrm{O} 3$ on tuber quality in potato (S. tuberosum L.). Agr. Ecosyst. Environ. 87, 273-285.

Drake, B.G.; M.A. González-Meler y S.P. Long. 1997. More efficient plants: a consequence of rising atmospheric $\mathrm{CO}_{2}$. Annu. Rev. Plant Physiol. Plant Mol. Biol. 48, 609-639.

Dwelle, R.B. 1990. Source/sink relationship during tuber growth. Amer. Pot. J. 67, 828-833.

Dwelle, R.B.; P. J. Hurley y J.J. Paveck. 1983. Photosynthesis and stomatal conductance of potato clones. Plant Physiol. 72, 172-176.

Estrada, R. N. 2000. La biodiversidad en el mejoramiento genético de la papa. Proinpa, Cip y Cid. La Paz, Bolivia.

Ewing, E. y P. Wareing. 1978. Shoot, stolon and tuber formation on potato (S. tuberosum L.) cuttings 
in response to photoperiod. Plant Physiol. 61, 348-353.

Farrar, J.F. y M.L. Williams. 1991. The effects of increased atmospheric carbon dioxide and temperature on carbon partitioning, source sink relations and respiration. Plant Cell Environ. 14, 819830.

FAO. 2009. FAO statistics. En: http://www.fao.org, 4 p.; consulta: septiembre de 2010.

Finnan, J.; A. Donnelly; J. Burke y M. Jones. 2002. The effects of elevated concentrations of carbon dioxide and ozone on potato (S. tuberosum L.) yield. Agr. Ecosyst. Environ. 88, 11-22.

Fulai, L.; A. Shahnazari; M. Andersen; S. Jacobsen y C. Jensen. 2006. Effects of deficit irrigation (DI) and partial root drying (PRD) on gas exchange, biomass partitioning, and water use efficiency in potato. Scientia Hort. 109,113-117.

Gawronska, H.; R.B. Dwelle; J.J. Pavek y P. Rowe. 1984. Partitioning of photoassimilates by for potato clones. Crop Sci. 24, 1031-1036.

Haverkort, A.J. 1990. Ecology of potato cropping systems in relation to latitude and altitude. Agr. Syst. 32, 251-272

Haverkort, A.J.; M. Van de Waart y K.B. A. Bodlaeader. 1990. The effect of early drought stress on numbers of tubers and stolons of potato in controlled and field conditions. Pot. Res. 33, 89-96.

Hawkes, J.G. 1997. A database for wild and cultivated potatoes. Euphytica 93, 155-161.

Hay, R. y A. Walker. 1989. An introduction to the physiology of crop yield. Second edition. Chapingo, México.

Hernández, G R. 1989. Nutrición mineral. Facultad de Ciencias Forestales, ULA, Mérida.

Hijmans, R. 2001. Global distribution of the potato crop. Amer. J. Pot. Res. 78(6), 403-412.

Högy, P. y A. Fangmeier. 2009. Atmospheric $\mathrm{CO}_{2}$ enrichment affects potatoes: 1. Aboveground biomass production and tuber yield. Eur. J. Agron. 30, 78-84.
Jackson, S.D. 1999. Multiple signaling pathways control tuber induction in potato. Plant Physiol. 119, $1-8$.

Jackson, S. y S. Prat. 1996. Control of tuberization in potato by gibberellins and phytochrome $B$. Physiol. Plant. 98, 407-412.

Karafyllidis, D.I.; N. Stavropoulos y D. Georgakis. 1996. The effect of water stress on the yielding capacity of potato crops and subsequent performance of seed tubers. Potato Res. 39, 153163.

Kimball, B.A. 1983. Carbon dioxide and agricultural yield: an assemblage and analysis of 430 prior observations. Agron. J. 75, 779-782.

Kooman, P. L.; M. Fahem; P. Tegera y A. Haverkort. 1996 Effects of climate on different potato genotypes 2. Dry matter allocation and duration of the growth cycle. Eur. J. Agron. 5, 207-217.

Ku, S.B.; G. Edwards y C. Tanner. 1977. Effects of light, carbon dioxide, and temperature on photosynthesis, oxygen inhibition of photosynthesis, and transpiration in $\mathrm{S}$. tuberosum. Plant Physiol. 59, 868-872.

Lafta, A.M y J. Lorenzen. 1995. Effect of high temperature on plant growth and carbohydrate metabolism in potato. Plant Physiol. 109, 637 643.

Larcher, W. 2003. Physiological plant ecology. Ecophysiology and stress physiology of functional groups. Forth edition. Springer Verlag, Berlín.

Lorenzen, J. y E. Ewing. 1992. Starch accumulation in leaves of potato (S. tuberosum L.) during the first 18 days of photoperiod treatment. Ann. Bot. 69, 481-485.

Lujan, L. 1991. Morfología, estructura y morfología de la planta de papa. Papa 2, 23-29.

Lujan, L. 1994. La ecología de la papa. Revista Papa 12. $1-36$

Mackerron, D. K y P. D. Waister. 1983. Light interception and dry matter accumulation in potato cultivars of contrasting habit. Potatoes 26,88 89 
Marschner, H. 1995. Mineral nutrition of higher plants. Academic Press. Second edition. New York, NY.

Meier, U. 2001. Estadios de las plantas mono y dicotiledóneas. 2a. ed. Centro Federal de Investigaciones Biológicas para Agricultura y Silvicultura, Braunschweig, Alemania.

Menzel, C.M. 1985. The control of storage organ formation in potato and other species. Field Crops 38, 527-537.

Ministerio de Agricultura y Desarrollo Rural. 2009. Secretaria Técnica Consejo Nacional de la Papa. Observatorio Agrocadenas Colombia: cuarto informe de coyuntura papa. En: http:// wWw.agrocadenas.gov.co/documentos/ Informes_coyuntura.htm\#C5; 8 p.; consulta: febrero de 2010

Nosberger, J. y E. Humpries. 1965. The influence of removing tuber on dry matter production and net assimilation rate of potato plants. Ann. Bot. $29,579-588$

Ortiz, E. y H. Hernández. 1986. Análisis de varianza fenotípica (densidad de población y niveles de fertilización) de los rendimientos y sus componentes en S. tuberosum Lin. Cultivos Tropicales 8(2), 65-71.

Pavlista, A. D. 2002. Environmental effects. Nebraska Potato Eyes 14, 1-4.

Piikki, K.; V. Vorne; K. Ojanpera y H. Pleijel. 2006. Impact of elevated $\mathrm{O}_{3}$ and $\mathrm{CO}_{2}$ exposure on potato ( $\mathrm{S}$. tuberosum L. Cv. Bintje) tuber macronutrients (N, P, K, Mg, Ca). Agr. Ecosyst. Environ. 118, $55-64$.

Ribó, M. 2004. Balance de macronutrientes y materia orgánica en el suelo de agrosistemas hortícolas con manejo integrado ecológico. Universidad de Valencia. Servicio de Publicaciones, Valencia, España.

Riesmeier, J.W.; L. Willmitzer y W.B. Frommer. 1994. Evidence for an essential role of the sucrose transporter in phloem loading and assimilate partitioning. Embo J. 13, 1-17.

Sarquis, J.I.; H. Gonzalez e I. Bernal-Lugo. 1996. Response of two potato clones (S. tuberosum L.) to contrasting temperature regimes in the field. Amer. Potato Res. 73, 285-300.
Schapendonk, A.; V. Oijen; P. Dijkstra; C. Pot; W. Jordi y G.M. Stoopen. 2000. Effects of elevated CO2 concentration on photosynthetic acclimation and productivity of two potato cultivars grown in open-top chambers. Aust. J. Plant Physiol. 27, 1119-1130

Sermet, O.; H.M. Caliskan y O. Caliska. 2005. Different irrigation methods and water stress effects on potato yield and yield components. Agric. Water Manage. 73, 73-86.

Snyder, E. 1989. Interactive effects of temperature, photoperiod and cultivar on tuberization of potato cuttings. HortScience 24, 336-338.

Sonnewald, W. 2001. Control of potato tuber sprouting Trends Plant Sci. 6(8), 333-335.

Spooner, D.; K. McLean; G. Ramsay; R. Waugh y G. Bryan. 2005. A single domestication for potato based on multilocus amplified fragment length polymorphism genotyping. Proc. Natl. Acad Sci. 102(41), 14694-14699.

Sukumaran, N.P. y C.D. Weiser. 1972. Freezing injury in potato leaves. Plant Physiol. 50, 564-567.

Taiz, L. y E. Zeiger. 2006. Plant physiology. Forth edition. Sinauer Associates Inc. Publ., Massachusetts, MA.

Tekalign, T. y P.S. Hammes. 2005. Growth and productivity of potato as influenced by cultivar and reproductive growth II. Growth analysis, tuber yield and quality. Scientia Hort. 105, 2944.

Thornton, M.K.; N.Y. Malik y R. Dwelle. 1996. Relationship between leaf gas exchange characteristics and productivity of potato clones grown at different temperatures. Amer. Potato J. 73, 63-77.

Tourneux, Ch.; A. Devaux; M.R. Camacho ; P. Mamani y J.F. Ledent 2003a. Effects of water shortage on six potato genotypes in the highlands of Bolivia (II): water relations, physiological parameters. Agronomie 23, 180-190.

Tourneux, Ch.; A. Devaux; M.R. Camacho ; P. Mamani y J.F. Ledent. 2003b. Effects of water shortage on six potato genotypes in the highlands of 
Bolivia (I): morphological parameters, growth and yield. Agronomie 23, 169-179.

Ulrich, A. 1993. Nutrition deficiencies and toxicities in crop plants. William F. Bennett, Minesota, MN.

Van-Delden, A.; A. Pecio y A. Haverkort 2000. Temperature Response of Early Foliar Expansion of Potato and Wheat. Ann. Bot. 86, 355-369.

Vreugdenhil, D.; J. Bradshaw; C. Gebhardt; F. Govers; D. MacKerron; A. Taylor y A. Heather. 2007. Potato biology and biotechnology: Advances and perspectives. Elsevier, Amsterdam.

Walworth, J.L y J.E. Muniz. 1993. A compendium of tissue nutrient concentrations for field grown potatoes. Amer. Pot. J. 70, 579-597.

WaddelI, J.T.; S. Gupta; J. Moncrief; H. Rosen y D. Steele. 2000. Irrigation and nitrogen-management impacts on nitrate leaching under potato. J. Environ. Qual. 29, 251-261.

Walker, T.S.; P.E. Schmiediche y R.J. Hijmans. 1999. World trends and patterns in the potato crop: An economic and geographic survey. Pot. Res. 42, 241-264.
Westermann, D.T. 2005. Nutritional Requirements of Potatoes. Amer. J. Pot. Res. 82, 301-307.

Wheeler, R.M. y T.W. Tibbits. 1986. Growth and tuberization of potato (Solanum tuberosum L.) under continuos light. Plant Phys. 80, 801-804.

Wright, A.J. y J. Brooks. 2002. Effect of windbreaks on potato production for the Atherton Tablelands of North Queensland. Aust. J. Exp. Agric. 42, 797-807.

Wu, J.; D. Wang y M. Bauer. 2007. Assessing broadband vegetation indices and Quick Bird data in estimating leaf area index of corn and potato canopies. Field Crops Res. 102, 33-42.

Yuan, F. y W. Bland. 2004. Light and temperature modulated expolinear growth model for potato (Solanum tuberosum L.). Agric. For. Meteor. 121, 141-151.

Yuan, B.Z.; S. Nishiyama y Y. Kang. 2003. Effects of different irrigation regimes on the growth and yield of dripirrigated potato. Agric. Water Manage. 63, 153-167. 